\title{
Educating the public on genetics and genomics: Carla Easter speaks to Ebony Torrington
}

\author{
Carla Easter ${ }^{1}$ \\ ${ }^{1}$ National Human Genome Research Institute, Education \& Community Involvement Branch, Bethesda, MD, USA \\ *Author for correspondence: easterc@mail.nih.gov
}

"Personally, I feel this work involves not only educating people but also bringing people closer to something that is very important to me - the awe of science and the understanding of how much it can do for us all."

First draft submitted: 20 March 2019; Accepted for publication: April 2019; Published online: 9 May 2019

Keywords: community engagement $\bullet$ genetics $\bullet$ genomic literacy $\bullet$ genomics $\bullet$ science education

Carla Easter is chief for the Education and Community Involvement Branch of the National Human Genome Research Institute (NHGRI). From 2003-2006, she was director of outreach for Washington University School of Medicine's Genome Sequencing Center. Before assuming her role as outreach director, Dr Easter was a research associate in the Department of Education at Washington University. She served as a project associate for the Quality Education for Minorities Network and conducted post-doctoral research at Washington University School of Medicine. Dr Easter earned her bachelor's degree in Microbiology from the University of California, Los Angeles and her doctoral in Biology with an emphasis on Molecular Genetics from the University of California, San Diego.

\section{You have always been heavily involved with science education \& outreach, now genomics} specifically. What drew you to this area of work?

As a graduate student, I undertook a lot of outreach work with students interested in becoming a part of the scientific community. It made me realize that access to opportunities to enter the field was not always balanced and was often unaffordable. Even as an undergraduate in science, I felt that certain students struggled more due to the fact that those early opportunities were often not available to them. I therefore believe that what drew me to my current work is the sense that everybody deserves an opportunity. I have always loved science and it has always made me incredibly sad that not everyone is afforded the opportunity to experience its beauty or engage in the joyful feeling of discovery. Regardless of whether a person intends to become a scientist, I believe everybody deserves that feeling. When I was a student, I did not anticipate that I would be where I am now but from an early stage, I made a conscious decision to do whatever I could to enhance opportunities for people to experience the joy of science. Personally, I feel this work involves not only educating people but also bringing people closer to something that is very important to me - the awe of science and the understanding of how much it can do for us all.

\section{What does your current role as chief of Education \& Community Involvement Branch at the} National Human Genome Research Institute entail?

Most of my work involves providing programming that might be used directly in the community and/or online. I am therefore responsible for a wide breadth of activities. We work with a lot of students, educators and community members but when it comes down to it, my focus is to enhance accessibility to information on genomics for people who want to either learn about it or even utilize it. My days generally involve a large number of meetings with a wide variety of stakeholders. I do, however, also work with a group of highly creative people within the branch that are always thinking about the next inspiring program that will help to educate people about genetics and genomics.

Future $\because$ Medicine 
I would therefore say that my job offers many opportunities to meet wonderful and exciting people and to work with communities that share a great amount of diversity.

\section{Why do you think educating the public in genomics specifically is so important?}

As we move forward, genomic information is going to become increasingly important to everyone. We are already seeing people engage in genetics through directed consumer testing such as ancestry testing. The idea of using genetic data has been around for a while, for example, in the form of family health history. People have been asking these questions and collecting this information for many years, while not necessarily realizing that they were actually collecting genetic data. I believe that healthcare is going to be the major place where this information will be important. I also imagine that it will offer a great deal of opportunity for entrepreneurship and for people that are thinking of unique ways in which genomic data could be utilized in the future. There are many important aspects to this and when you consider privacy specifically, given the data generated by various tests or the engagement with genetics and genomics, at some point we may have to make decisions about who uses the information and how they use it. It is therefore important for people to be educated on and have an understanding of the field.

\section{What about precision medicine? What work is being done at the governmental level to educate the public on this?}

We think about precision medicine in several different ways. The most obvious way right now is probably the 'All of Us' research program, supported by the National Institute of Health. The aim of this project is to bring together a cohort of one million individuals with very different backgrounds and then utilize this information in very precise ways. In addition to supporting the public's understanding of precision medicine, we also find ourselves very active in educating healthcare providers, to bring them up to speed on the utilization of genetic and genomic data so that they are more adept at applying this information to their patient populations. We are also interested in how we can prepare the users of that information and the people that are going to have to at some point explain how the genomic information is used. There are therefore many areas in which I can see precision medicine moving in to the mainstream.

\section{You were involved in the creation of the National Human Genome Research Institute/Smithsonian exhibition 'Genome: Unlocking Life's Code'. What role did you play in this \& what are the main goals of the exhibition?}

I was part of the National Human Genome Research Institute team that worked with the National Museum of Natural History to direct and develop the content and inform the look and 'feel' of the exhibition. The main goal of the exhibition was to introduce people to genomics and increase the public's literacy around this topic. Since the exhibit travels, we have been able to reach an abundance of communities, many that may not have otherwise had the opportunity to observe such a considerable amount of information on genetics and genomics in one place. We feel the exhibit lays a foundation for people to be introduced to genomics and hope it raises enthusiasm to learn more about this area of science. It is impressive that, although the exhibit has been traveling for a while, the interest and excitement around genomics does not appear to have waned. It has done a wonderful job in helping support genetic and genomic literacy. I am therefore very proud of my role and involvement in the development of this project. In addition, I am delighted that my branch was able to assist with the creation of the exhibit's website, 'Unlocking life's code', which we continue to maintain and update with new activities and resources.

\section{What do you feel are the biggest challenges for implementing genomic education into school curriculum? How do you \& the National Human Genome Research Institute plan to address these challenges?}

Key drivers of the US school curriculum include the standards maintained by educational institutions and what they must abide by. The challenge for us, in terms of getting genetics and genomics into elementary and secondary school curriculum, is the amount of teaching time available and whether the topic is considered necessary. A way in which we have dealt with this challenge is to reach out and work with educators to help them utilize the resources we create for classrooms. There is a great deal of work that still needs to be done and I do not think this is something we can do alone. We are therefore always looking for partners to work with whose expertise can help guide us and drive our efforts forward. 


\section{How would you like to see education for genomic \& precision medicine develop within the next $5-10$ years?}

I would like to see this information become highly accessible to people at all levels and from all backgrounds. In the next 5-10 years, I hope genomic education becomes as ubiquitous as some other areas of healthcare. Currently, you can go into an office building or pharmacy and take your blood pressure. I would like to see similar opportunities for people to learn about genetics and genomics. For example, it would be useful for people to be able to learn more about pharmacogenomics in their local pharmacy. We could tailor resources provided in each setting so that people can obtain relevant and specific information about genomics. Going forward, I therefore hope we can find unique and creative ways for people to gain this education in a comfortable setting.

\section{Are there any other important considerations that you wish to highlight?}

While I am very excited about the field's current trajectory, there are still many issues facing accessibility to this information that we are working on addressing. I believe, on many levels, the potential positives of enhancing knowledge about genomics far outweighs some of the negativity associated with it. In order for us to address some of the challenges and concerns however, we need to keep this dialogue open. Going forward, it will be important for conversations to take place between many communities, including educators, students, healthcare providers, politicians, community activists and anyone else involved in supporting communities. My hope is that such conversations become more public endeavors and a national dialogue is opened, where people can come together, feel comfortable asking questions without fear of judgement and can have an input on where we take genetics and genomics in the future.

Financial \& competing interests disclosure

The author has no relevant affiliations or financial involvement with any organization or entity with a financial interest in or financial conflict with the subject matter or materials discussed in the manuscript. This includes employment, consultancies, honoraria, stock ownership or options, expert testimony, grants or patents received or pending, or royalties.

No writing assistance was utilized in the production of this manuscript.

\section{Disclaimer}

The opinions expressed in this interview are those of the interviewee and do not necessarily reflect the views of Future Medicine Ltd.

\section{References}

1. National Human Geonome Research Institute (2019) www.genome.gov/staff/Carla-L-Easter-PhD

2. National Human Genome Research

Institute (2019). www.genome.gov/leadership-initiatives/Genomic-Literacy-Education-Engagement-Initiative

3. National Human Genome Research Institute (2019). www.genome.gov/about-nhgri/Division-of-Policy-Communications-andEducation/Education-and-Community-Involvement-Branch

4. National Human Genome Research Institute (2019). www.genome.gov/dna-day

5. Genome - Unlocking Life's Codes (2019). https://unlockinglifescode.org/ 
